\title{
Seismic investigation of the $\gamma$ Dor star KIC 6462033: The first results of Kepler and ground-based follow up observations
}

\author{
C. Ulusoy ${ }^{1}$, B. Ulaş ${ }^{2}$, M. Damasso ${ }^{3,4}$, A. Carbognani ${ }^{3}$, D. Cenadelli ${ }^{3}$, \\ I. Stateva ${ }^{5}$, I. Kh. Iliev ${ }^{5}$, and D. Dimitrov \\ ${ }^{1}$ College of Graduate Studies, University of South Africa, PO Box 392, UNISA 0003, Pretoria, \\ South Africa \\ email: cerenuastro@gmail.com \\ ${ }^{2}$ Department of Astronomy and Space Sciences, Faculty of Sciences, University of Ege, \\ Bornova, 35100, İzmir, Turkey \\ ${ }^{3}$ Astronomical Observatory of the Autonomous Region of the Aosta Valley (OAVdA) \\ Loc. Lignan 39, 11020 Nus (Aosta), Italy \\ ${ }^{4}$ Department of Physics and Astronomy, University of Padova, vicolo dell'Osservatorio 3, \\ I-35122 Padova, Italy \\ ${ }^{5}$ Institute of Astronomy with NAO, Bulgarian Academy of Sciences, blvd. Tsarigradsko \\ chaussee 72 , Sofia 1784 , Bulgaria
}

\begin{abstract}
Preliminary results on the analysis of the Kepler light curve and photometric groundbased time series of $\gamma$ Dor star KIC 6462033 (TYC 3144-646-1, $V=10.83, P=0.69686 \mathrm{~d}$ ) are presented in order to determine pulsation frequencies.
\end{abstract}

Keywords. stars: individual: KIC 6462033, stars: oscillations, stars: variables: $\gamma$ Dor

\section{Introduction}

$\gamma$ Dor variables are multiperiodic nonradial pulsators that oscillate in high-order gmodes with periods of the order of a day (Balona et al. 2011). Their position in the H-R diagram partially overlaps with the cool part of $\delta$ Sct instability strip. This means that stars showing both types of pulsations may exist (Uytterhoeven et al. 2011, Balona \& Dziembowski 2011). It should therefore be noted that searches for such objects are highly important for understanding the oscillation mechanisms of simultaneously excited p- and g-modes in a star.

\section{Ground-based and Kepler photometry}

Ground-based observations of KIC 6462033 were carried out at the Astronomical Observatory of the Autonomous Region of the Aosta Valley (OAVdA) between the Julian dates JD 2455740.4 - 2455776.5 . All data were obtained with the FLI PL3041-1-BB CCD camera attached to the $81-\mathrm{cm}$ telescope in the $U B V I$ photometric passbands. The observed light variation of the star in the $B$ filter is shown in Fig. 1.

In order to derive the frequency content of the variability of the star, we analyzed $K e$ pler short cadence (SC) data which consist of 38016 points taken in Kepler quarter Q3.3. The data were prepared for analysis using KEPCOTREND package (Fraquelli \& Thompson 2012, Christiansen et al. 2012, Ulusoy et al. 2013). 

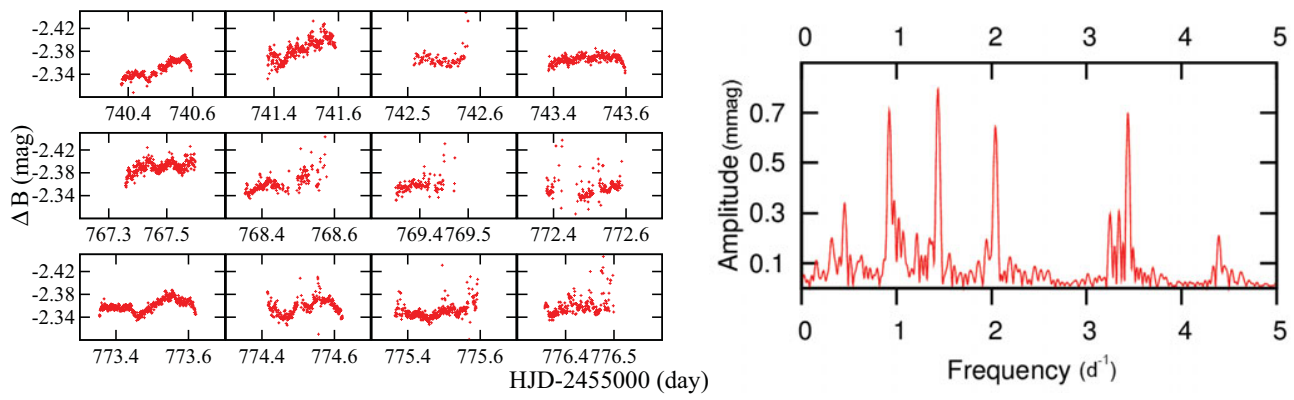

Figure 1. Left: B-filter light curves of KIC 6462033. Right: Fourier spectrum of SC Kepler data for KIC 6462033 .

\section{Fourier analysis}

We used PERIOD04 (Lenz \& Breger 2005) software for the analysis of both SC and ground-based time series. Following Breger et al. (2011), the signal to noise ratio $(\mathrm{S} / \mathrm{N})$ equal to 3.5 was adopted as a detection threshold. From the analysis of the Kepler data, we find that the light curve is dominated by four independent modes with frequencies $f_{1}=0.9242, f_{2}=1.4363, f_{3}=2.0409$ and $f_{4}=3.4257 \mathrm{~d}^{-1}$. Due to lack of data with sufficient quality, we were able to detect only the first two frequencies from the groundbased time series. The ground-based $B$-filter light curve and frequency spectrum of the Kepler data are shown in Fig. 1.

\section{Conclusions}

The star KIC 6462033 was recently classified as a $\gamma$ Dor-type star by Uytterhoeven et al. (2011). We have performed frequency analysis of the obtained ground-based data as well as the Kepler data for KIC 6462033. This is a first step of our scheduled study. We confirm that KIC 6462033 pulsates in the frequency range of $\gamma$ Dor type pulsators. In order to try seismic modeling, mode identification will be an essential requirement at least for a few frequencies. We expect that new photometric and spectroscopic observations will provide more data that can be used for this purpose.

\section{Acknowledgements}

CU sincerely thanks the South African National Research Foundation (NRF) for the award of NRF Multi-Wavelength Astronomy Research Programme (MWGR), Grant No: 86563 to Prof. L.L. Leeuw at UNISA, Reference: MWA1203150687.

\section{References}

Balona, L. A. \& Dziembowski, W. A. 2011, MNRAS, 417, 591

Balona, L. A., Guzik, J. A., Uytterhoeven, K., et al. 2011, MNRAS, 415, 3531

Breger, M., Balona, L., Lenz, P., et al. 2011, MNRAS, 414, 1721

Christiansen, J. L., Barclay, T., Jenkins, J. M., et al. 2012, Kepler Data Release 14 Notes (KSCI-19054-001)

Fraquelli, D. \& Thompson, S. E. 2012, Kepler Archive Manual (KDMC-10008-004)

Lenz, P. \& Breger, M. 2005, CoAst, 146, 53

Ulusoy, C., Ulaş, B., Gülmez, T., et al. 2013, MNRAS, 433, 394

Uytterhoeven, K., Moya, A., Grigahcène, A., et al. 2011, A\&A, 534, A125 